\title{
Regards
}

\section{Jean-Marie Legay, à la rencontre de la philosophie}

\author{
Anne-Françoise Schmid \\ Philosophe, INSA de Lyon, UMR5600 EVS-ITUS, 69621 Villeurbanne, France
}

$\mathrm{J}^{\prime}$ aimerais témoigner ${ }^{1}$ de notre rencontre, qui reste très vive dans ma mémoire. Jean-Marie Legay est venu me trouver alors que je signais un ouvrage dans une librairie lyonnaise. C'était en 1999. J'ai vu la porte s'ouvrir, d'une main ferme et tranquille, et dès que je l'ai aperçu, j'ai pensé que c'était une personne peu commune, habitée par la concentration des grands intellectuels.

Sa démarche était peu banale; non seulement le scientifique se déplaçait et se détournait de son chemin pour rencontrer une philosophe, mais, très rapidement, j'ai compris qu'il se comportait de façon peu habituelle dans les échanges entre disciplines. Il y a trente ans que je suis philosophe parmi les scientifiques, et mon expérience $\mathrm{m}$ 'a appris qu'ils m'abordent souvent pour m'apprendre ce qui se fait dans leur discipline : «voilà nos nouveautés, nos méthodes, voilà ce que vous pouvez en tirer ». Jean-Marie Legay s'est conduit tout à fait différemment, il a, dès l'entrée, non pas établi un dialogue simple, mais construit un espace dans lequel chacun pouvait déposer ce qui lui semblait important et dans lequel chacun pouvait laisser exister des interactions permettant le dialogue. C'est un geste rare, qui suppose une concentration, une maîtrise de sa discipline, une maîtrise telle, qu'elle induit le respect de la contribution des autres.

De cette rencontre ont découlé plusieurs choses, dans la même atmosphère d'échange. Tout d'abord, une demande à participer au comité de rédaction de la revue Natures Sciences Sociétés, qu'il avait fondée avec le sociologue Marcel Jollivet et le physicien Gérard Mégie en

Auteur correspondant : afschmid@free.fr

Anne-Françoise Schmid est également membre de la chaire TMCI de Mines ParisTech et chercheur associé aux Archives Poincaré, UMR 7117, à Nancy.

1 Une première version de ce témoignage a été prononcée par l'auteur lors de l'enterrement de Jean-Marie Legay le 13 avril 2012. Voir aussi, dans ce numéro et le précédent, les autres hommages à Jean-Marie Legay.
1993, à l'époque où toute l'équipe de la revue cherchait à « passer les frontières » disciplinaires. Deuxièmement, une proposition d'entretenir une correspondance sur les sujets qui nous paraissaient mériter un traitement interdisciplinaire ${ }^{2}$. Souvent, nous nous apportions les lettres aux réunions du comité de rédaction de NSS, pour ensuite les méditer chacun de notre côté. Enfin, l'invitation à participer à une commission "Analyse des systèmes complexes et interdisciplinarité » nommée par Gérard Mégie pour préparer un plan quadriennal du CNRS. On voit que 1'espace construit par Jean-Marie Legay et par notre collaboration a été déclinée de bien des façons, et qu'il a permis de créer un esprit d'entente et un commun intellectuel.

Un jour, je l'ai entendu dire à d'autres collègues : « Ce qu'il y a de certain, c'est qu'il ne faut plus parler seulement de la philosophie, mais des philosophies, et qu'il faut prendre au sérieux cette multiplicité ». Le commun était construit.

La construction d'un tel espace commun suppose un double regard ; tout d'abord, un regard très précis et pertinent sur le détail d'un problème. On sait comment Jean-Marie Legay a relié mathématiques et biologie, statistiques et biologie, et le rôle qu'il a joué dans la vaste question de la formalisation du vivant, comme en témoigne Franck Varenne dans Formaliser le vivant ${ }^{3}$. Mais $\mathrm{d}^{\prime}$ autre part, il avait aussi toujours un second regard qui lui permettait de placer le problème qu'il traitait dans un ensemble plus large. Donc, à la fois, un premier regard précis, qui fixe, et un second regard qui voit toute une

\footnotetext{
2 Cette correspondance a donné lieu à notre livre, pour expérimenter ce qu'un biologiste et une philosophe pouvaient construire ensemble : Legay, J.-M., Schmid, A.-F., 2004. Philosophie de l'interdisciplinarité. Correspondance (1999-2004) sur la recherche scientifique, la modélisation et les objets complexes, Paris, Pétra.

3 Varenne, F., 2010. Formaliser le vivant: lois, théories, modèles ? Paris, Hermann, chapitre 17.
} 
région. C'est ce qui donnait à Jean-Marie Legay son amour de la méthodologie, car la contemporanéité de ces deux regards n'est rendue possible que par l'existence d'une méthode qui édifie une sorte de cohérence ouverte.

La construction d'un tel espace était aussi le fait d'un savant qui sait que la généralisation de ses idées sur les sciences ne fait pas toute la science. N'oublions pas que le jour de ses vingt ans a eu lieu Nagasaki, c'était un choc qui montrait qu'une simple généralisation ne fait pas comprendre ce qui se passe. Ce qui lui importait, c'est la science. Il était comme un résistant, au sens premier et humain du terme, qui l'aime et ne l'abandonne pas. Dans les circonstances actuelles de la recherche où la vitesse et la compétition semblent importer pour son avancement, on entend souvent des chercheurs avouer ne pas reconnaître la pratique et l'idéal qu'ils avaient au début de leur carrière, voir disparaître la science et, finalement, concéder sa fin. Jean-Marie Legay n'était pas de ceux-là, il savait que son expérience ne la faisait pas toute et que l'on ne peut la généraliser ainsi sans construire des illusions. Il l'aimait sans douter.

Cet espace construit entre nous montrait aussi l'intérêt de Jean-Marie Legay, non seulement pour les grandes disciplines et les grandes analogies, mais également pour les petites, qui permettent des interactions et des échanges encore différents. Il a ouvert ainsi un champ qui reliait la science la plus théorique avec la pratique quotidienne. Je pense que nous devons nous inspirer de cet esprit, qui est celui qui permet de limiter les effets mimétiques de la compétition dans la recherche actuelle. Il nous faut revenir sur les liens entre l'idée que l'on dit universelle de science et le terrain dans chaque discipline et dans chaque lieu de recherche, pour stabiliser encore autrement une science que tant croient en danger.

Dans cette lutte quotidienne, Jean-Marie Legay donnait une grande importance à la culture scientifique qui lui apparaissait comme une nécessité, dans le "drame » actuel qui consiste dans une augmentation telle des connaissances que celles-ci deviennent ininterprétables. La culture scientifique donne une profondeur au double regard et permet de formuler des hypothèses pour proposer des interprétations.

Cette culture lui permettait de voir aussi les questions manquantes ; il a décliné avec moi bien des façons dont le concept d'homme était évité dans la biologie, voire dans la médecine, et il cherchait à reformuler les problèmes en essayant de pallier cette absence.

Tout cela montre l'engagement de Jean-Marie Legay. Pour lui, l'engagement et la science allaient ensemble. Lorsqu'il a débuté son métier de chercheur, commençait un débat assez vif sur les modèles et l'interdisciplinarité, débat qui remettait en cause, à la fois, sa posture scientifique et sa pratique interdisciplinaire. Il a défendu jusqu'au bout la valeur de sa démarche en affirmant que les modèles n'étaient pas des représentations. Cela permettait de réduire les contradictions que l'on voyait dans les projets interdisciplinaires. L'interdisciplinarité était alors critiquée comme produisant des contradictions que la théorie ne pouvait résoudre. On en imagine les conséquences épistémologiques, dont il était très conscient.

L'un de ses derniers livres, L'Expérience et le modèle a pour sous-titre Un discours sur la méthode ${ }^{4}$. Cela n'était pas naif. Jean-Marie Legay commence par placer Descartes en montrant que la situation scientifique dans laquelle il se trouvait le conduisait à postuler le simple, et qu'alors, c'était la meilleure solution. Il montre aussi la distance que nous avons prise avec la méthode expérimentale de Claude Bernard, voire avec les plans d'expérience de Ronald Fisher. Cette distance est nécessaire. En effet, si nous voulons vraiment rendre compte d'objets complexes, nous n'avons plus toujours la garantie de " groupes témoins ».

Cette épistémologie, il l'a mise d'ailleurs en pratique dans le grand projet de lutte contre la bilharziose lancé par la Délégation générale à la recherche scientifique et technique dans les années 1970. Ce projet est resté un modèle d'organisation de travail interdisciplinaire. JeanMarie Legay était capable sur un même objet, à la fois, de la pensée abstraite et de la réalisation, il était capable d'observer les vers à soie dans une tradition millénaire de recherche pour en construire finalement la modélisation indisciplinée ${ }^{5}$.

Il y a un sujet dont nous n'avons jamais parlé dans nos échanges, c'est de la politique. Il savait que je le savais engagé dans la Fédération mondiale des travailleurs scientifiques, que je connaissais le débat des modèles. Il savait que je voyais cet engagement comme condition nécessaire à son travail de chercheur, mais il ne me donnait jamais de détails. Il se conduisait comme un scientifique qui sait qu'il faut montrer qu'il y a un modèle sans croire à un modèle particulier, mais en tenant compte de leur multiplicité. Il montrait qu'il y avait un engagement, et les détails n'étaient pas ce qui importe. Par contre, il me disait parfois que certaines périodes de l'histoire le passionnaient. Il suffit de manifester l'engagement, non d'en parler.

Lorsque nous avons publié notre livre, Jean-Marie Legay m'a demandé d'y mettre un exergue tiré de La Rage de l'expression du poète Francis Ponge - toujours la rage et l'engagement. Le voici : "À propos de n'importe quoi, même d'un objet familier depuis des

\footnotetext{
4 Legay, J.-M., 1997. L'expérience et le modèle. Un discours sur la méthode, Paris, Inra Éditions.

5 Schmid, A.-F., 2002. Du ver à soie à la modélisation. Comment devient-on « indiscipliné »?, entretien avec Jean-Marie Legay, NSS, 10, 1, 59-63.
} 
millénaires à $1^{\prime}$ homme, il reste beaucoup de choses à dire ». C'est toujours le même regard. Il y a la banalité, le quotidien et, à la fois, l'invariant de tant de perspectives possibles, un multiple jamais fermé. C'est dans cet espace que nous convie la pensée de Jean-Marie Legay.
En tant que scientifiques et philosophes, nous ne pouvons que lui dire merci d'avoir existé et d'avoir pensé. À nous maintenant de continuer et de prolonger sa pensée et de la rendre plus lumineuse encore, c'est de notre responsabilité. 